\title{
Estrategias de cooperación académica para el desarrollo de la formación e investigación en Centroamérica y la Unión Europea
}

\author{
Academic cooperation strategies for the \\ development of training and research in Central \\ America and the European Union
}

Eugenio Trejos Benavides'

Antonio Juan Briones Peñalver ${ }^{2}$

Fecha de recepción: 20 de enero del 2012

Fecha de aprobación: 19 de mayo del 2012

Trejos, E; Briones, A. Estrategias de cooperación académica para el desarrollo de la formación e investigación en Centroamérica y la Unión Europea. Tecnología en Marcha. Vol. 25, Nº 6 Pág 4-I I

I Licenciado en Planificación Econoómica y Social, máster en administración pública. Candidato a doctor en Economía del desarrollo en la Universidad Autónoma de Madrid. Rector del Tecnológico de Costa Rica, periodo 2003-20ll. Profesor en la Escuela de Ciencias Sociales del Tecnológico de Costa Rica. Teléfono: 8840-4365 Correo: etrejos@itcr.ac.cr

2 Profesor, Universidad Politécnica de Cartagena Departamento de Economía de Empresa, Facultad de Ciencias de la Empresa, España. Teléfono: (34) 968325943 Correo electrónico: aj.briones@upct.es 


\section{Palabras clave}

Formación, investigación, agronegocios, emprendedor, economía social.

\section{Resumen}

El artículo resume las acciones de fortalecimiento institucional y proyectos de investigación para la formación e investigación de las capacidades de agronegocios en Costa Rica y la Región de Murcia.

La formación del profesorado, egresados y estudiantes; el conocimiento de las agro empresas y la emprendimientos en turismo rural, la economía social y el desarrollo de los agronegocios, la creación de spin-off, la logística y las operaciones de empresas hortofrutícolas, son algunos de los objetivos principales de estos proyectos de cooperación académica para el desarrollo de los países de Centroamérica y la Unión Europea.

Estos aspectos de la investigación llevarán a un mejor conocimiento de las zonas rurales y de las actividades agropecuarias para impulsar la consolidación en las regiones.

\section{Key words}

Training, research, agribusiness, entrepreneurship, social economy.

\begin{abstract}
The article summarizes the actions for the institutional strengthening and research projects for the training and research of the agribusiness capacities in Costa Rica and the Region of Murcia.
\end{abstract}

The training of teachers, graduates and students; the knowledge of agribusiness and rural tourism companies, social economy and the development of agribusiness, the creation of spin-off, logistics and the operations of horticultural companies are some of the main objectives of academic cooperation projects for the development of the Central American countries and the European Union.

These aspects of the research will lead to a better understanding of rural areas and agricultural activities to encourage the consolidation in the regions.

\section{Introducción}

La Universidad Politécnica de Cartagena (UPCT) y el Instituto Tecnológico de Costa Rica (ITCR, mejor conocido por las siglas TEC) han establecido relaciones de fortalecimiento institucional para la formación e investigación. Esta alianza tiene como propósito desarrollar estrategias de cooperación académica que posibiliten una mejora de las capacidades de los agronegocios de Costa Rica y de la Región de Murcia.

A finales de la década de los ochenta, la existencia de importantes desequilibrios socioeconómicos entre los diferentes territorios de la Unión Europea provocó que se replanteara la política regional comunitaria y la orientación de los Fondos Estructurales, en la búsqueda de la cohesión económica y social. Este contexto propicia la definición de iniciativas innovadoras dirigidas a corregir las defi- ciencias detectadas, así como a incentivar proyectos de desarrollo de la economía social para las áreas y los colectivos sociales más desfavorecidos, lo que ha tenido gran trascendencia para las áreas rurales y en diferentes sectores relacionados con la economía social de los países.

En este sentido, la dinámica de las micro, pequeñas y medianas empresas (MIPYMES) rurales tiende hacia su diversificación en actividades de turismo rural sostenible. Sobresalen en el caso de Costa Rica, los agronegocios de organizaciones de economía social enfocadas a buscar otras alternativas empresariales que fomenten el turismo rural sostenible.

La Región de Murcia cuenta con importantes atractivos patrimoniales para la actividad. De los resultados de los proyectos anteriores se ha observado que el análisis de las estrategias de agronegocios y el conocimiento de sus capacidades pueden ser 
de aplicación a las empresas turísticas de las zonas rurales tanto de Costa Rica como de España. La actividad turística en Costa Rica y Murcia tiene gran dinamismo tanto desde el ámbito público como privado en la modernización del sector, la mejora de la calidad de los servicios y su vertebración en la dinámica empresarial.

En la última década, las políticas y actuaciones encaminadas a la conservación y protección de la biodiversidad, a través del establecimiento de redes internacionales, estatales y regionales de espacios naturales protegidos están impulsando el desarrollo de actividades turísticas en sus territorios compatibles con la conservación de sus recursos naturales. Estos aspectos son de aplicación tanto en el caso de Costa Rica como en la Región de Murcia. Durante el 2010 y el 201 I se ha trabajado en la cooperación desde todos los aspectos antes mencionados, con lo cual se ha logrado:

I. La formación del profesorado, egresados, estudiantes, así como la consolidación del sector empresarial señalado para la investigación y conocimiento de los agronegocios turísticos, gestión del turismo rural sostenible y el agroturismo, en lo relativo a todos los aspectos conceptuales, la legislación aplicable a las organizaciones rurales, la creación de empresas, las estrategias directivas, el conocimiento de los sistemas de innovación a través de la generación de spin-off universitarias, la investigación en tecnologías agrarias y ambientales, las estrategias de diversificación de los agronegocios, la implantación de medidas de cooperación interempresarial para el fortalecimiento del tejido rural y autóctono de las regiones implicadas de Costa Rica y la Región de Murcia.

2. Determinación de agroempresas con potencial de intercambio entre Costa Rica y la Región de Murcia, con experiencia en sensibilidad ambiental y sostenibilidad económica-social que tengan emprendimientos en el sector del turismo rural sostenible.

3. Conocer la participación de todas las organizaciones, grupos de apoyo, ministerios y demás entidades gubernamentales que apoyan a las comunidades de proyectos de interés conjunto para España y Costa Rica.

4. Conocimiento de la economía social que facilite el emprendimiento en los agronegocios para generar oportunidades de desarrollo local en ambos países.

5. Analizar otros modelos de desarrollo empresarial en el ámbito de los agronegocios y por medio de las universidades involucradas (UPCTTEC), la creación de spin-off universitarias, el conocimiento de sus factores determinantes y la trasversalidad hacia otras formas empresariales relacionadas con la economía social.

6. Conocimiento de los aspectos logísticos de las empresas hortofrutícolas de ambos países para mejorar el abastecimiento y venta de productos y servicios, a través de la participación en otros proyectos relacionados con la Red Logística en el Sector Agroalimentario.

\section{Acciones de investigación y difusión de los resultados de la investigación preliminar}

Durante el 20 I I, de la misma forma que en el 20 I0, se ha seguido con la investigación en las entidades de la economía social de Murcia pertenecientes al sector hortofrutícola, industria agroalimentaria, productores de frutas y hortalizas y en general constitutivos de agronegocios en la Región. En el 2010 se logró tener el contacto con las Asociaciones más representativas de la Economía Social Murciana, Asociación de Empresas de la Economía Social de Murcia (AMUSAL), la Unión de Cooperativas de Trabajo Asociado (UCOMUR) y la Federación de Cooperativas Agrarias (FECOAM). A estas asociaciones se les administró un cuestionario de investigación en sus entidades asociadas con lo cual se ha obtenido información estadística de una muestra representativa de estas empresas e instituciones.

Asimismo, se han establecido contactos con la Plataforma Tecnológica de la Agricultura, así como intercambios con investigadores de Costa Rica del proyecto de Cooperación Internacional con Iberoamérica, pertenecientes a la Escuela de Ingeniería Agropecuaria y Administrativa del Tecnológico de Costa Rica (TEC) para obtener conocimiento sobre los agronegocios de la Región de Murcia. Por otra parte, se ha sumado a estos intercambios la Escuela de Ciencias Sociales del TEC, para contar con más conocimiento de la diversificación de oferta y demanda turística de Costa Rica así como de América Latina y Regiones del Caribe. También se ha podido participar en 
el proyector relacionado para el estudio de un "Prototipo de nodo red logística para sector hortofrutícola" (Fondo Tecnológico CDTI), con empresas contratantes representativas de la Región de Murcia con diferentes negocios en Iberoamérica como: Transportes Marcial, S.L. y Grupo Hortiberia, S.A.

En cuanto a la formación de profesorado en Costa Rica y en la Universidad Politécnica de Cartagena, se han dirigido dos trabajos de máster de estudiantes costarricenses y españoles. Uno de estos trabajo fue el realizado por Catalina María Morales Granados, titulado El turismo rural en la Región de Murcia y Costa Rica: un estudio comparativo. Otro trabajo fue el efectuado por María del Carmen Muñoz Medrano, denominado Gobierno corporativo en economía social, aportación de un modelo de gobierno. Además, se inició una tesis doctoral del funcionario del Tecnológico de Costa Rica, Mauricio Monge Agüero, titulada El emprendedor académico y la creación de spin-off universitario en Costa Rica.

Estas movilidades del profesorado investigador de Costa Rica se han programado mayoritariamente para planificación de las actividades de investigación, la recolección de datos primarios a través de la entrega de los cuestionarios estructurados. Ello ha permitido conocer de primera mano las posibilidades empresariales y de comercialización en común, a través de la visitas a lo largo de toda la Región para conocimiento de diferentes agronegocios y entidades de economía social, núcleos turísticos de la Comunidad Autónoma de Murcia y la industria mayoritariamente hortofrutícola y auxiliar naval de Cartagena.

Estas visitas se han realizado conjuntamente con la participación del profesorado de Costa Rica en determinados eventos académico tales como:

I. La presentación de libros donde participan investigadores de la Universidad Politécnica de Cartagena co sus trabajos de investigación:

- Equipos Docentes: Una nueva apuesta en el Espacio Europeo de Educación Superior (EEES)

- Programa de Redes de Investigación en Docencia Universitaria-Equipos Docentes

- Análisis estratégico para el desarrollo de la Mipyme en Iberoamérica

- Factores de éxito para favorecer la competitividad de la Pyme
- Informe Mipyme Iberoamérica, 2009, FAEDPYME

2. La presentación, por parte de Investigadores delTEC, del libro titulado Innovación y Desarrollo Integral de los Agronegocios en Costa Rica, en la Universidad Politécnica de Cartagena. Este libro es el resultado de un proyecto de investigación desarrollado en el 2010, y fue publicado por la Editorial Tecnológica de Costa Rica, Cartago, Costa Rica (ISBN: 978-9977-66-235-0).

3- La participación de investigadores de Costa Rica a determinados congresos internacionales con presentación de trabajos, como:

- Congreso Internacional de Innovación Docente 20II. Realizado en el Campus Mare Nostrum de la Universidad Politécnica de Cartagena.

- Fifth International Conference on Industrial Engineering and Industrial Management.

- Décimo quinto Congreso de Ingeniería de Organización.

- Twelveth European Conference on Creativity and Innovation (ECCl XII)- Portugal.

- First International Conference on Tourism and Management Studies, Escola Superior de Gestao, Hotelaria e Turismo, Faro, Portugal.

- Encuentro Internacional de Institutos y Centros de Investigación en Economía Social (ENUIES, 20II) Emprender desde la Innovación y la Transferencia de Conocimiento.

- IV Seminario Aula Abierta y Foro de Estudio de Seguridad y Defensa.

\section{Objetivos de la investigación}

Los objetivos de la investigación planteados y finalmente conseguidos son los siguientes:

1. Analizar la realidad de la Economía Social y las características fundamentales de las empresas de Economía Social relacionados con la agricultura en la Región de Murcia.

2. Conocer las iniciativas de emprendedores en el ámbito de las organizaciones de productores agrícolas en Economía Social de la Región de Murcia, la comercialización de sus productos a nivel nacional e internacional, la diversificación 
de sus actividades a través de los agronegocios y sus posibilidades en el sector turístico.

3. Estudiar los aspectos relacionados con la creación de empresas de Economía Social, y el perfil de los emprendedores, para determinar las medidas de gestión que pueden suponer una mejora en la excelencia, entre ellas: la responsabilidad social, la ética, la sostenibilidad, el medio ambiente, la innovación, la calidad, el conocimiento de los intangibles, los sistemas de información y el nivel de aplicación de TIC.

4. Establecer las estrategias de comunicación en las EES y las medidas de cooperación interempresarial que pueden ser más significativas para su desarrollo.

5. Divulgar los materiales de formación y resultados de la investigación en libros, así como la presentación de trabajos para evaluación en revistas y congresos de ámbito internacional.

Algunos de los objetivos proactivos en consecución del 2012 son:

a) Formar el profesorado para fomentar la investigación y la mejora de la oferta académica con Titulaciones Interuniversitarias a través de la participación de equipos docentes que propicien la innovación educativa en los diferentes ámbitos de las instituciones.

b) Proporcionar una plataforma académica interuniversitaria para el intercambio de las nuevas metodologías docentes, los sistemas de evaluación de competencias utilizados en los títulos del Espacio Europeo de Educación Superior (EEES) y promover tanto las TICs como las Titulaciones en Segunda Lengua en las Enseñanzas Universitarias y Secundarias.

c) Poner en valor los contenidos y resultados del conjunto de acciones desarrolladas en anteriores estudios de investigación entre el TEC de Costa Rica y la UPCT en España, en áreas de la economía, la responsabilidad social y las estrategias de cooperación en el ámbito de los agronegocios y las entidades de economía social.

d) Resaltar los logros académicos que han generado resultados con los proyectos PCI-AECID, a través de la creación de una red de atención de pequeños productores de agronegocios y entidades de economía social más represen- tativas en las Universidades de Centroamérica, particularmente en Costa Rica y focalizado a Nicaragua.

e) Dar a conocer los emprendimientos del sector del turismo rural y agroturismo de Costa Rica y Nicaragua,

f) Analizar los factores determinantes del gasto turístico en Centroamérica, y cuantificar la sensibilidad de la duración de la estancia a los cambios en variables relativas al perfil del turista y a los destinos.

g) Determinar las Mipymes de Centroamérica que pueden tener intercambio comercial con la Unión Europea, particularmente aquellas con experiencia en logística y transporte agroalimentario.

h) Analizar otros modelos de desarrollo empresarial en el ámbito de los agronegocios mediante las universidades involucradas (UPCT-TECUNAN-León), la creación de spin-off universitarios, el conocimiento de sus factores determinantes y la trasversalidad hacia otras formas empresariales relacionadas con la economía social.

i) Conocer los aspectos logísticos de las empresas hortofrutícolas de Costa Rica y Nicaragüa para mejorar el abastecimiento y venta de productos y servicios a través de la participación del profesorado de la UPCT en otros proyectos relacionados con la red logística en el sector agroalimentario.

La triangulación externa en la acción preparatoria que se está desarrollando actualmente está focalizada en Nicaragua para divulgar dentro del marco del Programa de Cooperación Interuniversitaria e Investigación Científica entre España e Iberoamérica los resultados del proyecto que ahora están justificados entre el TEC (Costa Rica) y la UPCT (España) .

\section{Impacto para la cooperación de Centroamérica con España}

Estos aspectos de la investigación llevarán a un mejor conocimiento de la multifuncionalidad de las zonas rurales y de las actividades agropecuarias para impulsar la consolidación en las regiones, con el desarrollo de actividades para: 
I. Intercambiar conocimientos adquiridos y la experiencia generada con los procesos de investigación.

2. Realizar encuentros técnicos científicos, cursos, jornadas empresariales, redes de intercambio, virtual web.

3. Promover el desarrollo de proyectos de $I+D+i$ entre empresas de la Región de Murcia y empresas de Costa Rica.

4. Realizar actividades de formación específica en Programas de Doctorado y Máster Oficiales Universitarios, en el campo de Gestión de Entidades de Economía Social y Gestión y Dirección de Empresas e Instituciones Turísticas.

5. Fortalecer la colaboración del TEC-UPCT en la Red Internacional de Investigadores en Pyme (REDIPYMES), creada por la Fundación para el Análisis de las Mipymes de Costa Rica y toda Iberoamérica.

Por otro lado, el grupo de investigación que inició con proyectos de cooperación en agronegocios ha desarrollado capacidades para la transferencia de conocimientos a las empresas hortofrutícolas, los agronegocios, la economía social y el turismo rural, para que puedan potenciar el desarrollo en las economías emergentes como la costarricense.

De esta manera, se han conseguido algunos de los objetivos de los convenios Marco y Específico de Colaboración Interuniversitaria entre el TEC y la UPCT, que han conllevado a nuevos acuerdos específicos para facilitar el acceso de profesores y estudiantes a programas universitarios .

El proyecto de fortalecimiento institucional aprobado para el 2012 ha sido generado gracias a las movilidades del profesorado durante el 201 I. Este proyecto posibilitará el establecimiento de vínculos estables de investigación y docencia entre los grupos de investigación del TEC y de la UPCT, con la inclusión de aspectos trasversales de interés para la cooperación internacional.

Uno de los objetivos de investigación iniciada en el 201 I es el análisis de los factores determinantes del gasto turístico de los extranjeros que visitan España y Costa Rica, mediante el uso de la información recopilada por la Encuesta de Gasto Turístico (EGATUR) del Instituto de Estudios Turísticos (IET) del Ministerio de Industria, Turismo y Comercio de España y la Encuesta Aérea de No Residentes del Instituto Costarricense de Turismo (ICT) de Costa Rica para turistas llegados por los diversos aeropuertos del país.

En una primera aproximación, tras realizar un estudio descriptivo y estadístico, se estimará, econométricamente, los determinantes del gasto de los turistas residentes en el extranjero cuyo destino es España y Costa Rica. Luego, se contrastarán y segmentarán estos resultados por destinos y actividades.

Ello permitirá precisar las variables socioeconómicas asociadas al mayor o menor gasto medio diario realizado por un turista durante su estancia, así como los efectos y sensibilidades que exiten sobre el gasto diario. Además, permitirá establecer otras características del viaje, tales como el número de pernoctaciones, las actividades realizadas, el tipo de alojamiento, la zona de visita, el origen del turista, entre otras.

De este proceso de investigación se ha derivado un trabajo final de la máster Catalina María Morales Granados, egresada del Tecnológico de Costa Rica y alumna del Máster de Gestión y Administración de Instituciones y Empresas Turísticas de la UPCT.

Por otra parte, uno de los objetivos alcanzados para el fortalecimiento institucional, a través de la investigación, ha sido el conocimiento de diferentes factores de éxito que puede proporcionar un auge en la ruralidad y en la organización de la población de Costa Rica, para mejorar los niveles de renta económica, la socialización del territorio, el intercambio cultural y estabilidad política. Ello permite establecer, debido a la proximidad, un paralelismo con losotros países de Centroamérica

En este sentido, se ha podido conformar un grupo de trabajo entre elTEC y la UPCT conjuntamente con el partenariado en la UNAN-León, donde participará un equipo de personal docente e investigador, con el exrector de la UPCT y el exrector del Tecnológico de Costa Rica, (coordinador del proyecto para la región centroamericana), más dos vicerrectores de la UPCT de Relaciones Internacionales y Planificación, múltiples centros de Ingeniería, Escuelas de Ciencias Sociales y Administración de Empresas y áreas de conocimiento, así como la dilatada experiencia del equipo investigador. Este entramado de relaciones 
conforma el siguiente equipo de trabajo de fortalecimiento institucional para la cooperación española:

Equipo de profesores e investigadores por España, Universidad Politécnica de Cartagena (UPCT)

- Coordinador: Antonio Juan Briones Peñalver, Facultad de Ciencias de la Empresa.

- Ramón Ruiz Merino, Vicerrector de Relaciones Internacionales y Campus Mare Nostrum.

- Beatriz Marín Noriega, Jefa del Servicio de Relaciones Internacionales de la UPCT; Lorenzo Ros McDonell, Escuela Superior de Ingeniería Industrial-Catedrático de Universidad.

- Andrés Artal Tur, Facultad de Ciencias de la Empresa-Titular de Universidad.

- Antonio García Sánchez, Facultad de Ciencias de la Empresa, Titular de Universidad.

- Elena de Lara Rey, Facultad de Ciencias de la Empresa, Titula de Escuela Universitaria.

- Isidoro Guzmán Raja, Facultad de Ciencias de la Empresa, Titular de Universidad.

- Carmen de Nieves Nieto, Escuela Superior de Ingeniería Industrial, P. Contratada Doctor.

- Eva Martínez Caro, Escuela Superior de Ingeniería Industrial, P. Contratada Doctor.

- Maria Victoria de la Fuente Aragón, Escuela Superior de Ingeniería Industrial, P. Contr.Doctor.

- Catalina María Morales Granados, Facultad de Ciencias de la Empresa - Máster en Gestión y Administración de Instituciones y Empresas Turísticas.

- María del Carmen Muñoz Medraño, Facultad de Ciencias de la Empresa- Doctorando.

Equipo de profesores e investigadores de Costa Rica, Instituto Tecnológico de Costa Rica (TEC).

- Coordinador: Eugenio Trejos Benavides, Escuela de Ciencias Sociales y exrector del TEC.

- Juan Rafael Hernández Navarro, Escuela de Ciencias Sociales-Profesor de Universidad.

- Mairim Carmona Pineda, Escuela de Ciencias Sociales- Profesora de Universidad.

- Pedro Martín Ramírez López, Escuela de Ciencias Sociales- Profesor y Doctorando.

- Marisela Bonilla Freer, Dirección de Cooperación de la Rectoría del TEC-TEC.
- Mauricio Monge Agüero, Técnico de Proyectos de Investigación del TEC-TEC.

- Óscar LópezVillegas, Coordinador de Proyectos de la Sede de San Carlos del TEC.

- Olger Murillo Bravo, Escuela de Agronomía en San Carlos-Profesor Universidad.

- Luis Gómez Gutiérrez, Ingeniería Electromecánica- Profesor de Universidad.

- Mario Chacón Rivas, Tecnológico Digital - TEC Digital.

Equipo de profesores y responsables de la Universidad Nacional de Nicaragua (UNAN-León).

- Coordinadora: MSc. Maritza Vargas, Vicerrectora de Relaciones Externas, y el profesorado:

- Óscar Martínez, Maestría en Turismo.

- Roberto Berrios, Doctor por la Universidad Autónoma de Madrid.

- Mario Talavera, Maestría en Computación.

- Francisco Valladares, Maestría en Administración.

El grupo de trabajo conformado proporcionaría a la Universidad Politécnica de Cartagena en España y a la Universidad de Alcalá de Henares su probable participación como socios en la Red Gira, (Grupo Interuniversitario de reflexion y apoyo al Desarrollo de Centroamérica) en la cual participan universidades estatales de países Centroamericanos, con el propósito de fortalecer su enfoque integracionista y la generación de acciones interuniversitarias en el ámbito diferencial Tecnológico y Desarrollo Técnico de Proyectos Científicos con la ejecución de iniciativas que permitan atender necesidades de sectores socieales y empresariales en Costa Rica, desde las universidades participantes en la Red y de manera pertinente para Centroamérica.

La Red GIRA constituye una plataforma de cooperación interuniversitaria solidaria entre universidades públicas de Centro América y España, la cual genera capacidades que permiten enfrentar juntas problemas del desarrollo humano sostenible de la Región, desde una perspectiva integracionista, de responsabilidad social y solidaridad.

\section{Conclusiones}

La época actual se caracteriza por la profunda transformación, económica, social, política y cultural, asociada a los procesos de globalización, y a los 
vertiginosos avances científicos y tecnológicos y, consecuentemente, a la transformación organizativa.

La irrupción tecnológica hacia el sector de la economía social, tercer sector o de servicios, ha posibilitado la construcción de un entramado de relaciones sinérgicas en ambientes de cooperación de los países de lberoamérica y España, a través del desarrollo de los agronegocios y la diversificación de la actividad en agroturismo. Los sectores terciarios tradicionales, se encuentran con nuevos formatos de empresas de carácter económico y social; cuentan con elementos competitivos de tecnologías emergentes que generan empleo en los territorios y un abanico de innovaciones vinculados a los procesos, los productos y los servicios ofrecidos.

El sector de la economía social configura una realidad empresarial formada por las entidades que comparten una serie de principios o características diferenciales enumeradas en el Proyecto de Fortalecimiento Institucional del Programa de Cooperación Interuniversitaria e Investigación Científica entre España e lberoamérica de la Agencia Española de Cooperación Internacional para el Desarrollo (AECID).

Entre esas características, las más importantes son la aplicación de principios de solidaridad y de responsabilidad, la democratización y la consecución de objetivos como el desarrollo sostenible, la equidad y la integración social.

Por otro lado, la trascendencia económica de las Entidades de Economía Social (EES) se manifiesta tanto en Iberoamérica. Así, Costa Rica es país de referencia, como lo es España -y en específico la Región de Murcia- para la Unión Europea, tanto por la participación en el Producto Interior Bruto y en la generación de empleo decente de las empresas que conforman este sector de economía protegida, como por su contribución al desarrollo de las políticas dirigidas al desarrollo y cohesión social y territorial.

Desde el Consejo Económico y Social de la Región de Murcia se anuncia que una de las principales razones para justificar el apoyo a las EES, radica en que estas entidades están caracterizadas por el compromiso de sus integrantes, quienes comparten singulares condiciones y brindan un valor añadido a la configuración del mercado de trabajo. Por esta razón, es conveniente implementar un conjunto de actuaciones que apoyen su actividad económica.
La actividad turística en Costa Rica y Murcia tiene gran dinamismo desde el ámbito tanto público como privado, en la modernización del sector, la mejora de la calidad de los servicios y su vertebración en la dinámica empresarial. La industria turística se está enfrentando a un nuevo entorno derivado de las transformaciones a las que se ha visto sometida. Este aspecto ha venido a modificar las tendencias del sector antes las demandas de un mercado que requiere concepciones más personalizadas y flexibles del producto o servicio turístico.

Las Tecnologías de Información y Comunicación en Turismo constituyen un paso hacia la modernización de este sector. Ante esta situación, las empresas con actividades turísticas necesitan orientar y centrar su modelo de gestión de negocio en las necesidades de sus clientes para poder afrontar los cambios y los retos planteados.

En este contexto, precisan más que nunca, disponer de información de calidad sobre la actividad desarrolladas, el mercado en el cual actúan y los agentes con los que se interrelacionan. Los aspectos señalados, convierten a la información y el conocimiento en este sentido como un activo para la gestión de la actividad económica de los territorios en cooperación.

Es en ese contexto que el equipo de investigación que inició con proyectos de cooperación entre el TEC y la UPCT en el campo de los agronegocios ha desarrollado capacidades para la transferencia de conocimientos a las empresas de economía social hortofrutícolas, de agronegocios y de turismo rural comunitario, para que estas puedan potenciar el desarrollo sostenible y la equidad y cohesión social en las economías emergentes como la costarricense.

De esta manera, se han alcanzado importantes objetivos trazados en los convenios Marco y Específico de Colaboración Interuniversitaria suscritos entre ambas instituciones, que han conllevado a nuevos acuerdos específicos para facilitar el acceso de profesores y estudiantes a programas universitarios

El proyecto de fortalecimiento institucional aprobado para el 2012 ha sido generado gracias a las movilidades del profesorado durante el 20I I. Este proyecto profundizará el establecimiento de vínculos estables de investigación y docencia entre los grupos de investigación del TEC y de la UPCT, con la inclusión de aspectos trasversales de interés para la cooperación internacional. 